\title{
Fluoroquinolone antibiotics and adverse events
}

\section{SUMMARY}

Fluoroquinolones are broad-spectrum antibiotics with good oral bioavailability. They are used for the treatment of a wide variety of infections, but there are restrictions on prescribing these drugs.

Epidemiological studies have reported an increased risk of rare adverse effects. These include tendinopathy and tendon rupture, peripheral neuropathy and aortic aneurysm.

Safe prescribing of fluroquinolones requires recognition of patients with risk factors for toxicity. Prompt drug discontinuation is recommended in the event of an adverse reaction.

Practising antimicrobial stewardship by prescribing fluoroquinolones only when alternative drugs are unavailable is also key to limiting adverse events and antibiotic resistance.

\section{Introduction}

Fluoroquinolones are broad-spectrum antibiotics with good oral bioavailability. Their indications include treatment of urinary tract infections, pneumonia, gastroenteritis and gonococcal infections. Worldwide, fluoroquinolone use is increasing and has been associated with rising rates of resistance. However, use remains relatively low in Australia compared to other countries due to restrictions placed on government-subsidised prescribing of fluoroquinolones.

In addition to the emergence of resistance, recent epidemiological studies have shown an association between fluoroquinolones and rare but significant adverse events. These include tendon rupture and aortic aneurysm and dissection.

\section{Mechanism of action and spectrum of activity}

The three systemic fluoroquinolones available for use in Australia are ciprofloxacin, norfloxacin and moxifloxacin. Additionally, ofloxacin is available for topical use in bacterial keratitis. Ciprofloxacin and norfloxacin have excellent activity against aerobic Gram-negative organisms, with ciprofloxacin being especially effective against Pseudomonas aeruginosa. Moxifloxacin has additional activity against anaerobes and Gram-positive organisms, particularly pneumococci, but lacks activity against $P$. aeruginosa. ${ }^{2}$ Fluoroquinolones exert antimicrobial effects by inhibiting bacterial topoisomerases II and IV. Antibiotic resistance arises from mutations in these target enzymes.

\section{Adverse effects}

The adverse effects of fluoroquinolones can be limited by restricting their use to infections that cannot easily be managed with other antibiotics.

\section{Tendinopathy}

Fluoroquinolones are associated with a two- to fourfold increased risk of acute tendinopathy (defined as pain or reduced function without rupture) and tendon rupture. ${ }^{3}$ The incidence of this adverse effect may be up to $2 \%$ in patients aged 65 years and above, compared with a background tendon rupture rate of approximately $0.9 \%$ in the general population. ${ }^{4,5}$

The onset of tendinopathy is highest within the first month after drug exposure. ${ }^{3}$ The Achilles tendon is most commonly affected, with severe and sudden onset pain being a characteristic clinical presentation. While optimal management of fluoroquinoloneassociated tendon disorders is unclear, prompt drug discontinuation is recommended alongside supportive measures such as analgesia and physiotherapy. The majority of patients (90\%) are managed nonoperatively with recovery taking a median of one month. There are long-term sequelae including difficulty walking, decreased range of motion and pain in up to $10 \%$ of patients. ${ }^{6}$

Risk factors for fluoroquinolone-associated tendinopathy are incompletely defined. Most studies report a pooled incidence from a range of fluoroquinolones including those currently unavailable within Australia. ${ }^{3,7}$ Older age and concomitant corticosteroid use are identified as risk factors. Corticosteroids are associated with up to a 14-fold

\section{Diva Baggio iD}

Advanced trainee

\section{Michelle R}

Ananda-Rajah iD

Infectious diseases and general medicine physician ${ }^{2,3}$

' Malignant Haematology and Stem Cell

Transplantation Service, Alfred Health

${ }^{2}$ General Medicine Unit, Alfred Health

${ }^{3}$ Central Clinical School, Monash University

Melbourne

\section{Keywords}

antimicrobial stewardship, ciprofloxacin

fluoroquinolones, moxifloxacin, norfloxacin, pharmacovigilance,

tendinopathy

Aust Prescr 2021:44:161-4

https://doi.org/10.18773/ austprescr.2021.035 
increased risk of rupture. ${ }^{3}$ An association with diabetes or renal failure is less clear.

The mechanism of tendinopathy is unknown. One study found that the risk increased with higher cumulative drug exposure, suggesting a dosedependent relationship. ${ }^{8}$ Collagen degradation due to upregulation of matrix metalloproteinases has also been proposed. ${ }^{3}$

\section{Aortic aneurysm and dissection}

The evidence associating fluoroquinolone use and aortic aneurysm and dissection is conflicting. A metaanalysis based on four observational studies has suggested a two- to threefold increased risk, with the number of fluoroquinolone courses needed to harm one patient being 1301. ${ }^{9}$ Observational studies, however, are affected by residual confounding and surveillance bias. To minimise differences in rates of imaging that may arise between fluoroquinoloneexposed and non-exposed patients, a case-control study of over one million US patients compared the risk of aortic aneurysm and dissection for specific antibiotic indications. Against azithromycin for pneumonia, the risk of aortic aneurysm and dissection was 2.5 times greater, but there was no risk difference when compared against trimethoprim-sulfamethoxazole for urinary tract infection. The absolute rate of aortic aneurysm and dissection was also very low at less than $0.1 \%$. The authors concluded that the benefits of fluoroquinolones may outweigh a rare potential risk of aortic aneurysm and dissection in some patients. ${ }^{10}$

In contrast, another study found no difference in the risk of aortic aneurysm and dissection between fluoroquinolones and other antibiotics typically used for severe infections (such as amoxicillin-clavulanate or cephalosporins). However, there was an increased risk across all types of infection including pneumonia, genitourinary, intra-abdominal, and soft tissue and bone infections. The authors suggested that infection severity may therefore confound the observed association between fluoroquinolone exposure and aortic disease, particularly as fluoroquinolones are often used for more serious infections and in older patients." Alternatively, a surveillance bias toward imaging patients with severe infection may have strengthened the observed association between sepsis and aortic aneurysm and dissection.

A recent retrospective study reported the risk of rupture, surgery and death only in patients admitted with aortic aneurysm or dissection, ${ }^{12}$ thus reducing the risk of surveillance bias. It found a 1.8-fold increased risk of 'aortic death' in patients who had taken fluoroquinolones. However, the comparator group was amoxicillin and not a broader spectrum drug, possibly confounding the range of infections for which the two groups of patients were treated.

When aortic aneurysm and dissection are detected, patients should stop the drug immediately and be referred for appropriate observation and surgical intervention. Particular care should be taken in patients with a family history of aneurysm, preexisting aortic aneurysm, or the presence of other risk factors including Marfan's syndrome or other connective tissue diseases. There is an updated warning in the Australian and international product information for fluoroquinolone antibiotics..$^{13}$

\section{Peripheral neuropathy}

A limited number of observational studies suggest that fluoroquinolones increase the risk of peripheral neuropathy, manifesting as numbness or pain, by up to 1.5 -fold. ${ }^{14-16}$ However, the occurrence of neuropathy in fluoroquinolone-exposed patients is still rare, with an absolute risk increase in a large database study of just $0.02 \%$ per year among all patients, and $0.04 \%$ per year in those aged 60 years or above. ${ }^{14}$ Risk factors for fluoroquinolone-associated neuropathy include increased body mass index, as well as other known causes of neuropathy such as amyloidosis, alcohol abuse, shingles, and Sjögren syndrome. However, the association between neuropathy and fluoroquinolone use, in the database study, remained even in a subgroup analysis excluding patients with these underlying conditions. ${ }^{14}$

The mechanism of neuropathy remains undefined, with case reports describing evidence of small fibre damage on nerve biopsy. ${ }^{16}$ It is unclear if symptoms are reversible after stopping the drug.

\section{Retinal detachment}

Evidence for an association between fluoroquinolone use and retinal detachment is conflicting. While a Canadian cohort study reported an up to 4.5-fold increased risk of retinal detachment with current fluoroquinolone use, ${ }^{17}$ most subsequent studies, including two recent meta-analyses, ${ }^{18,19}$ have found no increased risk. One explanation for the findings of the Canadian study was the presence of an older cohort (mean age 61 years) including patients postcataract surgery which is a known risk factor for retinal detachment. ${ }^{17}$

\section{QTC prolongation and cardiac arrhythmia}

The absolute risk of torsades de pointes with fluoroquinolone use is low, equating to 160 additional serious arrhythmias per 1,000,000 antibiotic courses. However, the risk can be increased by hypokalaemia, hypomagnesaemia and drugs that prolong the QTC interval on the ECG. ${ }^{20}$ There is a particular risk 
associated with moxifloxacin. This drug probably has twice the risk of arrhythmia compared to ciprofloxacin and levofloxacin. ${ }^{21}$

QTC prolongation occurs due to blockade of cardiac delayed rectifier potassium channels. This leads to prolongation of the action potential. ${ }^{22}$

\section{Gastrointestinal effects}

Nausea, vomiting, diarrhoea and taste disturbance have been reported to occur in up to $20 \%$ of patients treated with fluoroquinolones. ${ }^{23}$ Like all antibiotics, fluoroquinolones carry a risk of Clostridium difficile infection. Fluoroquinolone-resistant $C$. difficile infections have emerged in response to increased fluoroquinolone prescribing. ${ }^{24}$

Hepatotoxicity with elevation in transaminases is a class effect of fluoroquinolones. Severe hepatotoxicity with acute liver failure is extremely rare, with the exception of trovafloxacin which was withdrawn from the market. ${ }^{25}$

\section{Hyperglycaemia and hypoglycaemia}

Observational studies have reported increased risks for hyperglycaemia and hypoglycaemia with fluoroquinolones. ${ }^{26-28}$ The risk of hyperglycaemia (2.5-fold) and hypoglycaemia (2.1-fold) is higher with moxifloxacin than with ciprofloxacin (1.9- and 1.5-fold) after adjustment for relevant baseline factors such as diabetic treatment. ${ }^{28}$

The dysglycaemia is thought to be due to sulfonylurea-like effects on the ATP-sensitive potassium channels of pancreatic islet cells. ${ }^{26}$

\section{Central nervous system effects}

Fluoroquinolones have been associated with adverse effects on the central nervous system, including psychiatric adverse reactions. These effects have been included in a boxed warning, on the product labels, by the US Food and Drug Administration. The reported adverse reactions include agitation tremor, hallucinations, psychosis and convulsions.

\section{Conclusion}

In Australia, fluoroquinolone prescribing is restricted to infections resistant to all other recommended drugs. Serious adverse effects are rare but significant, and include tendinopathy, aortopathy, neuropathy, arrhythmia, hypoglycaemia and hyperglycaemia. Prescribers should be aware of the risk factors for fluoroquinolone toxicity including patients over 60 years and patients with comorbidities or interacting drugs.

Patients should remain vigilant for symptoms such as tendon or abdominal pain and report these promptly. Appropriate patient education and prompt drug discontinuation in the event of an adverse reaction are important considerations when prescribing fluoroquinolones. $<$

Conflicts of interest: Michelle Ananda-Rajah has received speaker fees and competitive research funding from MSD.

\section{REFERENCES}

1. Cheng AC, Turnidge J, Collignon P, Looke D, Barton M, Gottlieb T. Control of fluoroquinolone resistance through successful regulation, Australia. Emerg Infect Dis 2012;18:1453-60. https://doi.org/10.3201/eid1809.111515

2. Andriole VT. The quinolones: past, present, and future. Clin Infect Dis 2005;41 Suppl 2:S113-9. https://doi. org/10.1086/42805

3. Alves C, Mendes D, Marques FB. Fluoroquinolones and the risk of tendon injury: a systematic review and meta-analysis. Eur J Clin Pharmacol 2019;75:1431-43. https://doi.org/10.1007/ s00228-019-02713-1

4. Yasui Y, Tonogai I, Rosenbaum AJ, Shimozono Y, Kawano H, Kennedy JG. The risk of achilles tendon rupture in the patients with achilles tendinopathy: healthcare database analysis in the United States. BioMed Res Int 2017;2017:7021862. https://doi.org/10.1155/2017/7021862

5. Daneman N, Lu H, Redelmeier DA. Fluoroquinolones and collagen associated severe adverse events: a longitudinal cohort study. BMJ Open 2015;5:e010077. https://doi.org/ 10.1136/bmjopen-2015-010077

6. Khaliq Y, Zhanel GG. Fluoroquinolone-associated tendinopathy: a critical review of the literature. Clin Infect Dis 2003;36:1404-10. https://doi.org/10.1086/375078

7. Stephenson AL, Wu W, Cortes D, Rochon PA. Tendon injury and fluoroquinolone use: a systematic review. Drug Saf 2013;36:709-21. https://doi.org/10.1007/s40264-013-0089-8

8. Morales DR, Slattery J, Pacurariu A, Pinheiro L, McGettigan P, Kurz X. Relative and absolute risk of tendon rupture with fluoroquinolone and concomitant fluoroquinolone/ corticosteroid therapy: population-based nested casecontrol study. Clin Drug Investig 2019·39:205-13. https://doi.org/10.1007/s40261-018-0729-y
9. Dai XC, Yang XX, Ma L, Tang GM, Pan YY, Hu HL. Relationship between fluoroquinolones and the risk of aortic diseases: a meta-analysis of observational studies. BMC Cardiovasc Disord 2020;20:49. https://doi.org/10.1186/ s12872-020-01354-y

10. Gopalakrishnan C, Bykov K, Fischer MA, Connolly JG, Gagne JJ, Fralick M. Association of fluoroquinolones with the risk of aortic aneurysm or aortic dissection. JAMA Intern Med 2020;180:1596-605. https://doi.org/ 10.1001/jamainternmed.2020.4199

11. Dong $\mathrm{YH}$, Chang $\mathrm{CH}$, Wang JL, Wu LC, Lin JW, Toh S. Association of infections and use of fluoroquinolones with the risk of aortic aneurysm or aortic dissection. JAMA Intern Med 2020;180:1587-95. https://doi.org/10.1001/ jamainternmed.2020.4192

12. Chen SW, Chan YH, Chien-Chia Wu V, Cheng YT, Chen DY, Lin CP, et al. Effects of fluoroquinolones on outcomes of patients with aortic dissection or aneurysm. J Am Coll Cardiol 2021;77:1875-87. https://doi.org/10.1016/ j.jacc.2021.02.047

13. US Food and Drug Administration. FDA warns about increased risk of ruptures or tears in the aorta blood vessel with fluoroquinolone antibiotics in certain patients. FDA Drug Safety Communication. Updated 2018 Dec 21. https://www.fda.gov/drugs/drug-safety-and-availability/ fda-warns-about-increased-risk-ruptures-or-tears-aortablood-vessel-fluoroquinolone-antibiotics [cited $2021 \mathrm{Jul} 5$ ]

14. Morales D, Pacurariu A, Slattery J, Pinheiro L, McGettigan P, Kurz X. Association between peripheral neuropathy and exposure to oral fluoroquinolone or amoxicillin-clavulanate therapy. JAMA Neurol 2019;76:827-33. https://doi.org/ 10.1001/jamaneurol.2019.0887 
15. Etminan M, Brophy JM, Samii A. Oral fluoroquinolone use and risk of peripheral neuropathy: a pharmacoepidemiologic study. Neurology 2014;83:1261-3. https://doi.org/10.1212/ WNL.0000000000000846

16. Cheng JZ, Sodhi M, Etminan M, Carleton BC.

Fluoroquinolone use and risk of carpal tunnel syndrome: a pharmacoepidemiologic study. Clin Infect Dis 2017:65:684-6. https://doi.org/10.1093/cid/cix362

17. Etminan M, Forooghian F, Brophy JM, Bird ST, Maberley D. Oral fluoroquinolones and the risk of retinal detachment. JAMA 2012;307:1414-9. https://doi.org/10.1001/jama.2012.383

18. Chui CS, Wong IC, Wong LY, Chan EW. Association between oral fluoroquinolone use and the development of retinal detachment: a systematic review and meta-analysis of observational studies. J Antimicrob Chemother 2015;70:971-8. https://doi.org/10.1093/jac/dku507

19. Alves C, Penedones A, Mendes D, Batel Marques F. A systematic review and meta-analysis of the association between systemic fluoroquinolones and retinal detachment. Acta Ophthalmol 2016;94:e251-9. https://doi.org/10.1111/ aos.12931

20. Liu X, Ma J, Huang L, Zhu W, Yuan P, Wan R, et al. Fluoroquinolones increase the risk of serious arrhythmias: a systematic review and meta-analysis. Medicine (Baltimore) 2017;96:e8273. https://doi.org/10.1097/ MD 0000000000008273

21. Gorelik E, Masarwa R, Perlman A, Rotshild V, Abbasi M, Muszkat $M$, et al. Fluoroquinolones and cardiovascular risk: a systematic review, meta-analysis and network meta-analysis. Drug Saf 2019;42:529-38. https://doi.org/10.1007/ s40264-018-0751-2
22. Alexandrou AJ, Duncan RS, Sullivan A, Hancox JC, Leishman DJ, Witchel HJ, et al. Mechanism of hERG $\mathrm{K}+$ channel blockade by the fluoroquinolone antibiotic moxifloxacin. Br J Pharmacol 2006;147:905-16. https://doi.org/10.1038/sj.bjp.0706678

23. Owens RC Jr, Ambrose PG. Antimicrobial safety: focus on fluoroquinolones. Clin Infect Dis 2005;41 Suppl 2:S144-57. https://doi.org/10.1086/428055

24. Vardakas KZ, Trigkidis KK, Boukouvala E, Falagas ME. Clostridium difficile infection following systemic antibiotic administration in randomised controlled trials: a systematic review and meta-analysis. Int J Antimicrob Agents 2016;48:1-10 https://doi.org/10.1016/j.ijantimicag.2016.03.008

25. Andrade RJ, Tulkens PM. Hepatic safety of antibiotics used in primary care. J Antimicrob Chemother 2011;66:1431-46. https://doi.org/10.1093/jac/dkr159

26. Park-Wyllie LY, Juurlink DN, Kopp A, Shah BR, Stukel TA, Stumpo C, et al. Outpatient gatifloxacin therapy and dysglycemia in older adults. N Engl J Med 2006;354:1352-61. https://doi.org/10.1056/NEJMoa055191

27. Aspinall SL, Good CB, Jiang R, McCarren M, Dong D, Cunningham FE. Severe dysglycemia with the fluoroquinolones: a class effect? Clin Infect Dis 2009;49:402-8. https://doi.org/ $10.1086 / 600294$

28. Chou HW, Wang JL, Chang CH, Lee JJ, Shau WY, Lai MS. Risk of severe dysglycemia among diabetic patients receiving levofloxacin, ciprofloxacin, or moxifloxacin in Taiwan. Clin Infect Dis 2013:57:971-80. https://doi.org/ $10.1093 / \mathrm{cid} /$ cit439 